\title{
Inventario multitemporal, análisis de susceptibilidad y estimación de volumen de deslizamientos en el flanco SW del volcán Pico de Orizaba, Puebla-Veracruz
}

\author{
Gabriel Legorreta Paulín ${ }^{1, *}$, Marcus Bursik², Pouget Solene ${ }^{2}$, José Lugo Hubp ${ }^{3}$, \\ Luis Mario Paredes Mejía ${ }^{4}$, Fernando Aceves Quesada ${ }^{3}$ \\ ${ }^{1}$ Instituto de Geografía, Laboratorio de Análisis Geoespacial, Ciudad Universitaria, Del. Coyoacán, 04510, México D.F. \\ ${ }^{2}$ Department of Geology, University at Buffalo, SUNY Buffalo, NY 14260. \\ ${ }^{3}$ Instituto de Geografía, Departamento de Geografía Física, Universidad Nacional Autónoma de México, Ciudad Universitaria, Del. \\ Coyoacán, 04510, México D.F. \\ ${ }^{4}$ California Department of Transportation (CALTRANS).
}

*legorretag@hotmail.com; legorretag@igg.unam.mx

\begin{abstract}
Resumen
Este artículo provee un panorama general de la investigación en curso del Instituto de Geografía de la UNAM, que busca crear una metodología integral para el desarrollo de cartografía de inventarios multitemporales, de susceptibilidad y estimación de volúmenes de deslizamientos mediante el uso de Sistemas de Información Geográfica (SIG). La metodología se establece para terrenos volcánicos, donde deslizamientos superficiales y flujos de escombros son frecuentes a lo largo de las laderas y los sistemas fluviales de los volcanes. La cuenca del Río Chiquito-Barranca del Muerto en el flanco suroeste del volcán Pico de Orizaba se ha seleccionado como caso de estudio para caracterizar las áreas que son propensas a la inestabilidad de las laderas en terrenos volcánicos. La ocurrencia de deslizamientos se determinó a través de un inventario de deslizamientos creado a partir de fotos aéreas multitemporales y trabajo de campo. En este estudio se modela (simula) la susceptibilidad a deslizamientos usando un modelo cartográfico-hidrológico (Stability Index MAPping (SINMAP)) y un modelo estadístico (Regresión Logística Múltiple (RLM)). Ambos modelos están integrados dentro de la aplicación LOGISNET (desarrollado mediante el uso de macros (AML) del sistema de información geográfica (SIG) ArcInfo Workstation. Su implementación mostró que ambos modelos poseen un grado moderado de concordancia con el inventario de deslizamientos, prefiriéndose la RLM para futuros análisis por su flexibilidad de incluir otras variables. Para la estimación de volúmenes de materiales aportados por los deslizamientos a los cauces, se seleccionaron los deslizamientos superficiales. El área y volumen de deslizamientos superficiales individuales fueron obtenidos en campo y a través de la base de datos del inventario de deslizamientos, para establecer una relación empírica: área-volumen. La relación se utilizó para el uso de una ley de potencias con la que se estimó el volumen total de deslizamientos de tierra en el área de estudio. Los resultados son importantes para comprender la evolución a largo plazo del sistema fluvial en el flanco suroeste del volcán Pico de Orizaba, y apoyar el desarrollo de una metodología útil para la elaboración de inventarios, el análisis de la susceptibilidad y estimación de volúmenes de deslizamientos en terrenos volcánicos.
\end{abstract}

Palabras clave: Deslizamientos, SIG, análisis de la susceptibilidad, volcán Pico de Orizaba.

\begin{abstract}
This paper provides an overview of the ongoing research from the Institute of Geography, UNAM, that seeks to create a comprehensive methodology for the development of a multi-temporal mapping inventory, susceptibility, and landslide volume estimation by using Geographic Information Systems (GIS). The methodology is established for volcanic terrains where shallow landslides and
\end{abstract}


debris flows are common along the slopes and fluvial systems of volcanoes. The Rio-Chiquito-Barranca del Muerto watershed on the southwestern flank of Pico de Orizaba volcano was selected as a case study to characterize the areas that are prone to slope instability in volcanic terrains. Landslide occurrence was determined through a landslide inventory created from multitemporal aerial photos and fieldwork. In this study, landslide susceptibility was modeled using a cartographic-hydrologic model (Stability Index Mapping SINMAP) and a statistical model (Multiple Logistic Regression - MLR). Both models were integrated within LOGISNET (developed in Arc Macro Language (AML) using ArcInfo GIS software). The implementation showed that both models have a moderate degree of agreement with the landslide inventory. MLR was preferred for further analysis because of its flexibility to include other variables. Shallow landslides were selected to estimate the volume of material delivered by the landslides to the main stream channel. The area and volume of individual shallow landslides were obtained in the field and through the landslide inventory database to establish an empirical area-volume relationship. The relationship was used to establish a power law in order to estimate the total landslide volume delivered. The results are important in understanding the long-term evolution of the fluvial system on the southwestern flank of Pico de Orizaba, and support the development of a methodology for landslide inventories, landslide susceptibility, and landslide volume estimation in volcanic terrains.

Keywords: Landslides, SIG, susceptibility analysis, Pico de Orizaba volcano.

\section{Introducción}

En ambientes volcánicos, y especialmente en los grandes estratovolcanes, se generan deslizamientos pequeños, de menos de $102 \mathrm{~m}^{3}$, aunque son peligrosos (Montgomery y Dietrich, 1994; Pack et al., 2001), mismos que afectan las laderas volcánicas e incrementan el poder destructivo de los flujos de escombros y lodo a lo largo de los cauces fluviales, lo cual a su vez aumenta el riesgo para los asentamientos y la actividad humana. Es el caso del volcán Pico de Orizaba y de muchos de los grandes estratovolcanes de México (Siebe et al., 1996; Capra et al., 2002; CarrascoNúñez et al., 2006; Evans et al., 2006). Sin embargo, y a pesar de la importancia de evaluar dicho proceso, hay pocos inventarios de deslizamientos, y por consiguiente, hay también una carencia de análisis de susceptibilidad (entendido como la propensión de un área a ser afectada por deslizamientos en función del grado inherente de estabilidad de la pendiente) y de evaluación de materiales aportados por los deslizamientos a los sistemas fluviales. Este es el caso del volcán Pico de Orizaba, la montaña más alta de México (5675 m s.n.m.), el cual tiene un gran potencial para producir deslizamientos y flujos de escombros, debido a la existencia de depósitos piroclásticos, de caída y de rocas altamente meteorizadas que están sujetas a condiciones de alta precipitación estacional. Así por ejemplo, en el año 2003, vidas y bienes económicos se perdieron en la población de Balastrera (un asentamiento localizado en el valle inferior del Río Chiquito-Barranca del Muerto, en el flanco SO del volcán), debido a lluvias torrenciales que incrementaron el aporte de materiales de deslizamientos a un flujo de escombros. Durante el evento, un poliducto fue destruido por el flujo de escombros. A raíz de este suceso, la compañía estatal Petróleos Mexicanos (PEMEX), encargada del mantenimiento de esta red de tuberías, construyó una serie de muros de retención y gaviones a lo largo del valle inferior del Río Chiquito-Barranca del
Muerto para proteger sus instalaciones. Sin embargo, ningún inventario de deslizamientos, ni medida preventiva se llevó a cabo en la parte media y alta de la cuenca. Lo anterior trajo como consecuencia que en el periodo de lluvias de 2011, los muros de retención y gaviones fueran destruidos por deslizamientos y por un flujo de escombros que se desarrollaron en la parte superior y media de la cuenca. Además, pocos estudios se han realizado en el uso y comparación sistemática de diferentes modelos de susceptibilidad de deslizamientos (Morrissey et al., 2001; Chinnayakanahalli et al., 2003; Haneberg, 2005; LegorretaPaulín y Bursik, 2008), así como para analizar el aporte de materiales por parte de los procesos gravitacionales. Como resultado, el uso de estos enfoques y aplicaciones en los SIG para la predicción de deslizamientos sin el conocimiento de sus ventajas y desventajas puede inducir a un mal uso de los modelos y a obtener conclusiones erróneas. Con base en lo anterior, el objetivo principal de este trabajo es desarrollar una metodología para la elaboración de un inventario multitemporal, el análisis de susceptibilidad y la estimación de volúmenes de deslizamientos que apoyen a las autoridades gubernamentales y al público en general en la planeación, prevención y mitigación de riesgos de procesos gravitacionales.El inventario fue elaborado a partir de dos series de fotos aéreas digitales y trabajo de campo. Los procesos de remoción en masa fueron digitalizados en el SIG, y una base de datos geoespacial se desarrolló para una mejor descripción. El trabajo de campo y la fotointerpretación permitió la representación cartográfica de 442 eventos. El análisis de la relación de distribución espacial entre los deslizamientos con este inventario y la geología reveló que los procesos gravitacionales son más abundantes en la parte media-alta de la cuenca, donde predominan depósitos piroclásticos y de caída. Asimismo se analizó la susceptibilidadpor deslizamientos de tierra mediante un modelo cartográfico-hidrológico (SINMAP) y un modelo estadístico (Regresión Logística Múltiple 
(RLM)). Ambos modelos están implementados en la aplicación de LOGISNET (desarrollado mediante el uso de macros (AML) del programa de sistema de información geográfica (SIG) ArcInfo Workstation (Legorreta-Paulín y Bursik, 2008, 2009). Los modelos fueron evaluados y comparados usando una tabla de contingencia que muestra la cantidad de sobreposición entre el inventario y los modelos. Para la estimación de volúmenes aportados por los deslizamientos al sistema fluvial, se midieron valores de área y volumen de 102 deslizamientos superficiales durante el trabajo de campo. Dichas medidas de área y volumen de deslizamientos se utilizaron para establecer una relación empírica que tomó la forma de una ley de potencias. La relación empírica se utilizó para estimar el volumen potencial total del material aportado por todos los deslizamientos superficiales del río principal en la cuenca.

\section{Antecedentes}

Los inventarios de deslizamientos históricos o multitemporales constituyen la base para los análisis de la susceptibilidad, del peligro y del riesgo por deslizamientos, así como de la estimación de volúmenes de materiales aportados por dichos procesos. Su representación cartográfica y el análisis se llevaron a cabo e incorporaron en los SIG (Peark et al., 2005; Washington State Department of Natural Resources, 2006; Kalderon-Asael et al., 2008; Hervás y Bobrowsky, 2009; Guzzetti et al., 2009; Blahut et al., 2010; Wenkey et al., 2011). Por su parte, la evaluación de la susceptibilidad a deslizamientos y su zonificación se ha abordado de manera heurística, estadística o determinística (Dai et al., 2002; Clerici et al., 2002; Zhou et al., 2003; Metternicht et al., 2005; Demoulin y Chung, 2007; Castellanos-Abella y Van Westen, 2008). Asimismo se han hecho esfuerzos para probar y validar los modelos de susceptibilidad de deslizamientos de tierras (Montgomery y Dietrich, 1994; Pack et al., 1997, 1998, 2001; Morrissey et al., 2001; Dietrich et al., 2001; Borga et al., 2002a, 2002b; Zaitchik et al., 2003; Wawer y Nowocień, 2003; Chinnayakanahalli et al., 2003; Lan et al., 2004; Winsemius et al., 2005; Legorreta-Paulín y Bursik, 2008). A nivel mundial, dos enfoques han sido usados para predecir deslizamientos potenciales en zonas con escasa información espacial: el Índice de Mapeo de Estabilidad (Stability Index MAPping (SINMAP)) y la Regresión Logística Múltiple (RLM). El primero de ellos, fue implementado con un enfoque cartográfico/hidrológico para expresar la estabilidad de la pendiente del terreno en términos de un índice de estabilidad (Pack et al., 1998). Este índice expresa la estabilidad del terreno en una clasificación de seis categorías. El índice es calculado mediante la combinación del factor de seguridad del modelo de estabilidad con pendiente infinita (Hammond et al., 1992) y la teoría de un modelo hidrológico (Beven y Kirkby, 1979; O’Loughlin, 1986). El segundo modelo, la RLM, estima la probabilidad de deslizamientos entre 0 y 1 usando una función logística que considera la contribución de una serie de variables. Esta función tiene una forma sigmoidal que puede ser interpretada como de susceptibilidad mínima de un área a sufrir deslizamientos cuando la función tiene valores bajos y ésta se mantiene como de baja susceptibilidad hasta que cierto umbral es alcanzado, entonces la probabilidad de deslizamientos aumenta rápidamente conforme los valores de la función se incrementan (Kleinbaum y Klein, 2002). Ambos modelos definen bien las zonas que intuitivamente parecen ser susceptibles a deslizamientos (Morrissey et al., 2001; Ohlmacher y Davis, 2003; Yesilnacar y Topal, 2005; Can et al., 2005; Ayalew y Yamagishi, 2005; Meisina y Scarabelli, 2007; Deb y El-Kadi, 2009; Bai et al., 2010; Van Den Eeckhaunt et al., 2005). Estos dos modelos fueron adaptados en el sistema, LOGISNET, mediante el uso de Arc Macro Language (AML) en el SIG ArcInfo. El sistema se ha utilizado para organizar, adaptar, comparar y visualizar los resultados de RLM y SINMAP (LegorretaPaulín y Bursik, 2009). Sin embargo, la evaluación de los modelos ha sido difícil de realizar debido a la complejidad de problemas concomitantes naturales y técnicos (Zaitchik et al., 2003; Legorreta-Paulín et al., 2010). La evaluación es especialmente difícil en los modelos diseñados para la detección o predicción de deslizamientos superficiales o someros. Esto es debido a que las dimensiones de los deslizamientos superficiales son pequeñas en comparación con otros tipos de deslizamientos (Can et al., 2005). En cuanto a la estimación de volúmenes de material aportado a los ríos por parte de los deslizamientos, éstos han sido llevados a cabo mediante el uso de funciones que usan la ley de potencias, a fin de enlazar las medidas de área de las zonas afectadas por los deslizamientos y el volumen desplazado (Peark et al., 2005; Kalderon-Asael et al., 2008; Guzzetti et al., 2009; Wenkey et al., 2011).

En México, el Centro Nacional de Prevención de Desastres (CENAPRED) ha compilado conceptos básicos de la clasificación de deslizamientos, mecanismos de activación, criterios, consideraciones y el análisis para el reconocimiento del riesgo a deslizamientos, en forma de guías para la preparación de atlas estatales y municipales de riesgos de deslizamientos (Centro Nacional de Prevención de Desastres, 2004). Así mismo, muchos de los estudios y las aplicaciones de procesos gravitacionales basados en los SIG han sido usadas creativamente y de muchas maneras para representar y evaluar la estabilidad de las laderas. Cada uno de ellos con sus propias ventajas y limitaciones en la evaluación de deslizamientos a escala local o regional, se han implementado utilizando criterios determinísticos, estadísticos, heurísticos o simplemente elaborando un inventario (Capra et al., 2006; Capra y Lugo-Hubp, 2006; García-Palomo et al., 2006; Pérez- Gutiérrez, 2007). En el estado de Veracruz, un atlas de peligros geológicos e hidrometeorológicos está siendo elaborado por la Secretaría de Protección Civil del estado de Veracruz, en colaboración con otras agencias del gobierno federal y estatal. Este 
atlas cuenta con un marco general para la evaluación de los riesgos a deslizamiento a nivel estatal y municipal (Comunicación personal: Secretaría de Protección Civil, 2010). En el volcán Pico de Orizaba, los estudios geológicos de los últimos treinta años se han enfocado en establecer los posibles mecanismos y estilos eruptivos que explican la actual morfología y el peligro potencial de los eventos volcánicos y de colapso de sus flancos (De la Cruz-Reyna y Carrasco-Núñez, 2002; Macías, 2005). Con base en los estudios geológicos previos, varios modelos y métodos usando SIG y Percepción Remota (PR), han sido propuestos a escala regional o local para explicar y evaluar los peligros de deslizamientos y flujos de escombros catastróficos (Sheridan et al., 2002; Zimbelman et al., 2004; Centro Nacional de Prevención de Desastres, 2004; Concha-Dimas et al., 2005; Rodríguez et al., 2006; Hubbard, 2001; Hubbard et al., 2007; Secretaría de Protección Civil, 2010). En el área de estudio se realizó en 2003 un análisis general de las condiciones climatológicas y geológicas que dispararon un flujo de baja concentración en el valle inferior del río Chiquito Muerto-Barranca (Rodríguez et al., 2006, 2011), sin embargo no se elaboró el inventario de deslizamientos o la susceptibilidad de la cuenca.

\section{3. Área de estudio}

La cuenca del río Chiquito-Barranca del Muerto se encuentra entre $\operatorname{los} 18^{\circ} 48^{\prime} 28^{\prime \prime}$ y $19^{\circ} 01^{\prime} 49^{\prime \prime}$ de latitud norte y $97^{\circ} 10^{\prime} 5^{\prime \prime}$ y $97^{\circ} 16^{\prime} 12^{\prime \prime}$ de longitud oeste, dentro de los estados de Puebla y Veracruz, República de México, y cubre un área de $111 \mathrm{~km}^{2}$ (Figura 1). La cuenca se localiza en el flanco suroeste del volcán Pico de Orizaba, en la parte oriental de la provincia fisiográfica del Eje Volcánico Transmexicano. El río es afluente del Río Blanco, que desemboca en el Golfo de México. El área es afectada de manera importante por procesos gravitacionales, flujos de escombros y lodo debido a la combinación de varios factores, tales como la alta precipitación, cambio en el uso del suelo causados por la tala intensiva, el alto grado de meteorización de las rocas localizadas a gran elevación y en laderas de fuerte pendiente.

En el área de estudio el relieve es montañoso y escarpado, con elevaciones de 1340 a 5675 m.s.n.m. y pendientes desde los $3^{\circ}$ (valles interiores y llanuras) a más de $60^{\circ}$ (terreno montañoso). Las pendientes de las laderas son controladas por la litología, por ejemplo, las lutitas débilmente consolidadas y calizas presentan pendientes de hasta $32^{\circ}$. Laderas con pendiente fuerte $\left(32^{\circ}-45^{\circ}\right)$ se encuentran en capas de conglomerados cementados, aglomerados y lahares. Los flujos de lava constituyen laderas con pendientes mayores a $45^{\circ}$. La precipitación media anual es de $1000-1100 \mathrm{~mm} /$ año por arriba de los 4000 m.s.n.m. y de 927 mm/año por debajo de 1500 m.s.n.m. (Palacios et al., 1999). La mayoría de la precipitación se produce entre mayo y noviembre. El cauce del río ChiquitoBarranca del Muerto erosiona un basamento cretácico de caliza y lutita fuertemente intemperizado, disectado y plegado (cubre el 29.7 \% del área), que ha sido cubierto por lavas, flujos piroclásticos y depósitos de caída del Neógeno (60.3 \%) y del Plioceno-Cuaternario (2.4 \%), así como por aluvión (7.6\%) (Figura 2).

\section{Metodología}

La metodología comprende tres etapas principales. Etapa 1: Elaboración de un inventario de deslizamientos históricos o multitemporal (pasos 1 a 6 en la Figura 3); Etapa 2: Análisis de la susceptibilidad para la cuenca y evaluación de modelos (paso 7, Figura 3), y la Etapa 3: Cálculo del volumen aportado por los deslizamientos al cauce principal (paso 8, Figura 3).

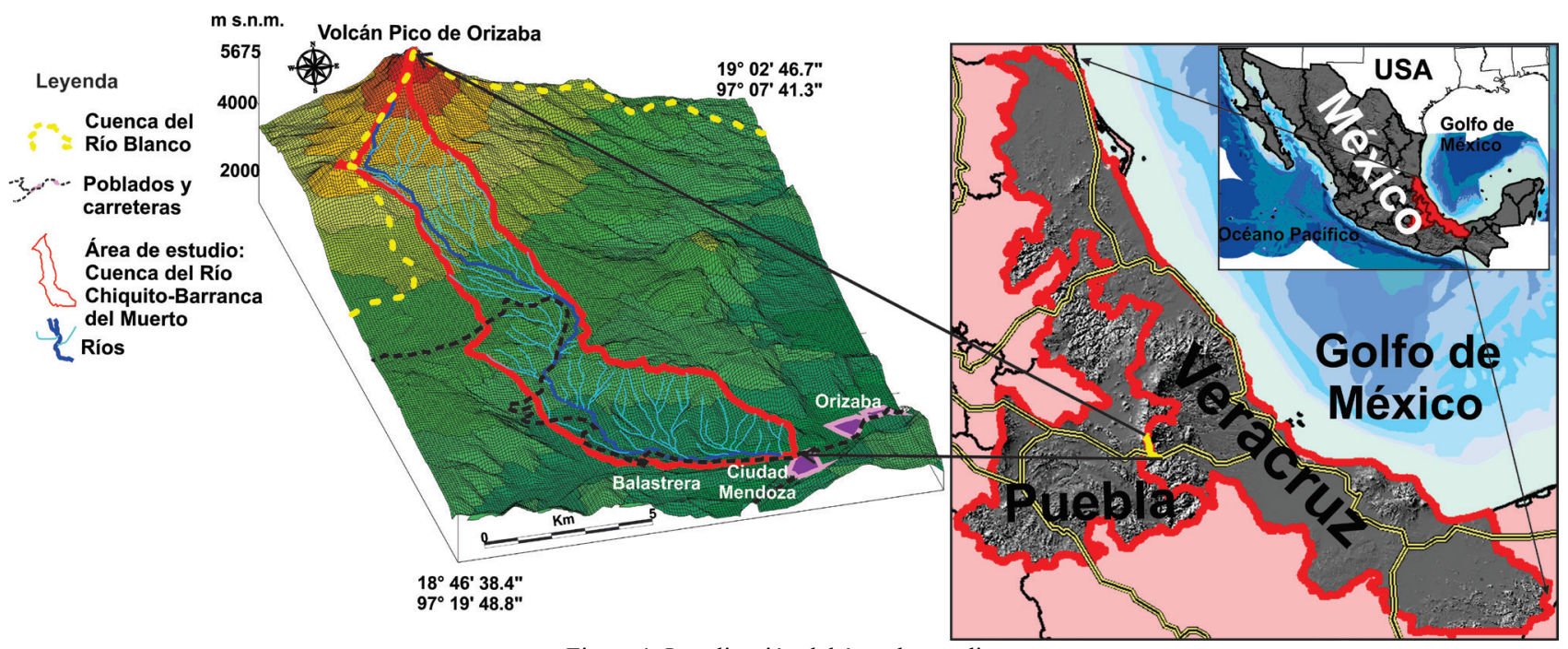

Figura 1. Localización del área de estudio. 


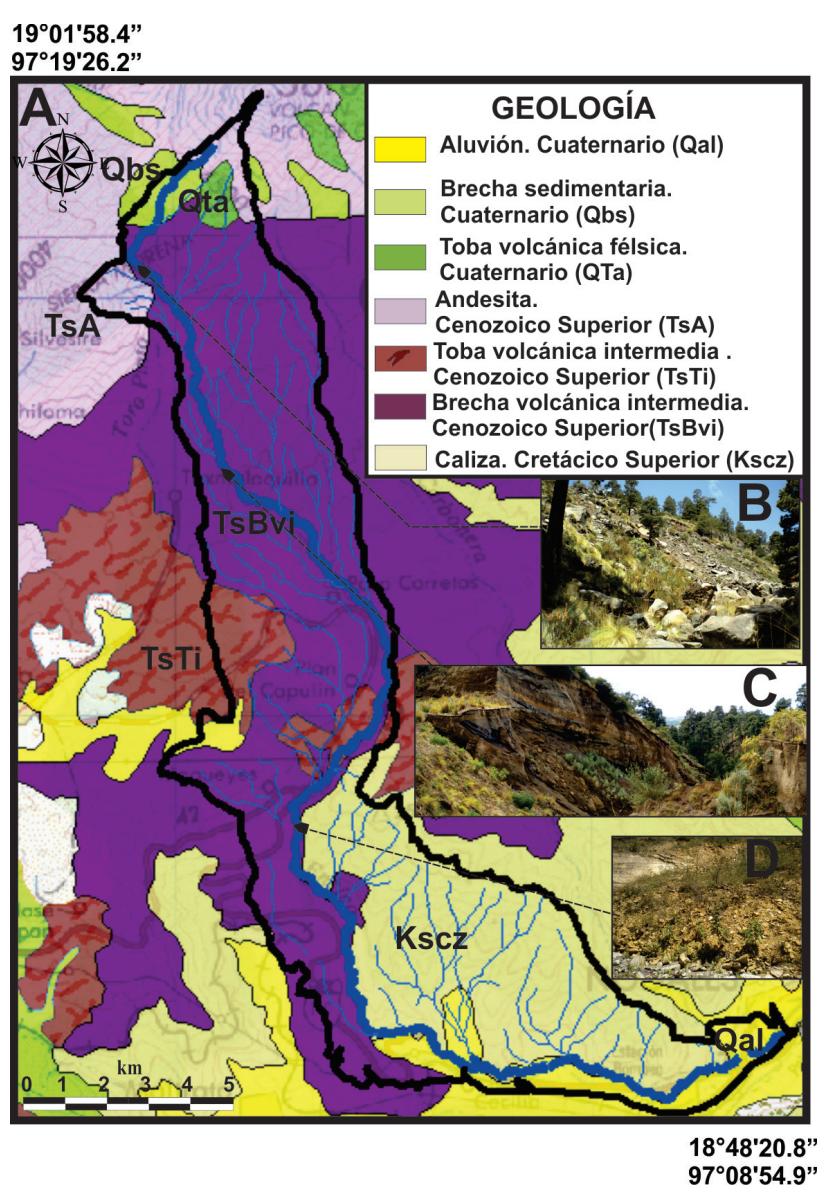

Figura 2. a) Mapa geológico; b) Caída de rocas en coladas de lava; c) Deslizamiento de asentamiento profundo; d) Deslizamiento de flujo de tierra.

Para la primera etapa, el área de estudio fue seleccionada (paso 1, Figura 3) como piloto por ser representativa de una cuenca ubicada en relieve volcánico y contar con las condiciones fisiográficas que propician continuos deslizamientos superficiales. En el paso 2, se recopiló la información preexistente para definir los procesos de remoción en masa ocurridos en la cuenca y evitar la duplicidad o enfoques de otros trabajos. Se colectaron los mapas impresos: topográficos y geológicos a escala 1:50000, y de uso del suelo, clima e hidrología a escala 1:250000. Todos los mapas impresos se convirtieron a un formato 'raster' de $10 \mathrm{~m}$ de resolución; fueron georeferenciados e incorporados como una capa más de información dentro del SIG y se incluyó un modelo digital de elevación (MDE) de $10 \mathrm{~m}$ de resolución creado por el Instituto Nacional de Estadística y Geografía (INEGI) a partir de la carta topográfica 1:50000. También se incluyeron los mapas temáticos derivados de éste: de pendientes, de curvatura del terreno y de área de contribución de escurrimiento superficial. La carta topográfica junto con las capas digitales fueron utilizadas para ayudar en la fotointerpretación de deslizamientos y para verificar y trazar los polígonos de deslizamientos en el SIG.
Derivado de la compilación de información y en paralelo con la selección e incorporación de capas en el SIG (paso 4, Figura 3), se seleccionaron los conjuntos temporales y representativos más completos de fotos aéreas a usar (paso 3, Figura 3). La selección estuvo basada en la disponibilidad de fotos aéreas. Los deslizamientos fueron cartografiados a partir de dos series de fotos aéreas y trabajo de campo para crear un solo mapa histórico de inventario de deslizamientos (paso 5 en Figura 3 y Figura 4a). Las fotos aéreas cubren un período de 14 años. Una serie es de 1994, a escala 1:20000, y otra de 2008, a escala 1:10000. Durante la interpretación de las fotos aéreas y el trabajo de campo, los deslizamientos fueron cartografiados y clasificados en deslizamientos superficiales, de escombros, y de asentamiento profundo, flujos de escombros, flujos de tierra y caída de rocas, de acuerdo con los criterios del grupo de Washington State Department of Natural Resources (DNR), Forest Practices Division (2006). Esta clasificación simplifica el sistema de Cruden y Varnes (1996) y Wieczorek (1984) al listar sólo la clasificación primaria de un deslizamiento de tierra determinado. Esto la hace práctica y fácil de usar en campo y en gabinete para la preparación del inventario de deslizamientos de un área de estudio (Pringle y Brunego, 2004; Sharikahn y Walsh, 2007). Todos los deslizamientos de tierra fueron digitalizados en el SIG de ArcInfo, y se construyó la base de datos geoespacial de deslizamientos. Los atributos pertinentes de los deslizamientos se colectaron en el SIG. Estos incluyen: 1) tipo deslizamiento, 2) nivel de certeza de la observación, 3 ) fecha de identificación en la foto aérea, 4) tamaño del deslizamiento, 5) grado de actividad del deslizamiento, 6) la parte del deslizamiento que se describe (la cabecera, la zona de evacuación, el depósito), 7) forma de la pendiente (cóncava, plana, convexa), 8) gradiente topográfico, 9) gradiente topográfico tomado del mapa topográfico, 10) uso del suelo, 11) elevación donde comienza el deslizamiento, 12) número de identificación de la fotografía aérea, 13) área del deslizamiento, 14) volumen del deslizamiento, y 15) los comentarios de investigador. En paralelo con la interpretación de fotografías aéreas y la cartografía de deslizamientos, se realizó el trabajo de campo (paso 6, Figura 3), a fin de obtener una visión real de la cuenca y de los tipos y procesos gravitacionales existentes. El porcentaje de verificación de deslizamientos en campo fue de $37 \%$, lo que permitió aumentar la confianza en la evaluación de estos procesos realizado con las fotos aéreas.

Para la segunda etapa, la susceptibilidad a deslizamientos en las laderas fue analizada usando RLM y SINMAP. SINMAP expresa la estabilidad del terreno utilizando seis clases subjetivas: de $0>$ SI (SI es el índice de estabilidad) ("inestable"), $0.5>$ SI > 0.0 ("del umbral más alto de estabilidad"), $1.0>$ SI $>0.5$ ("del umbral más bajo de estabilidad"), $1.25>\mathrm{SI}>1.0$ ("casi estable"), $1.5>\mathrm{SI}>1.25$ ("moderadamente estable"), y SI > 1.5 ("estable") (Figura4b) (Pack et al., 1997, 1998). Las propiedades geotécnicas requeridas para correr el modelo se establecieron con base en valores obtenidos en campo y valores publicados (Tabla 
Metodología para el inventario, la cartografía de susceptibilidad y estimación de volúmenes de deslizamientos usando SIG

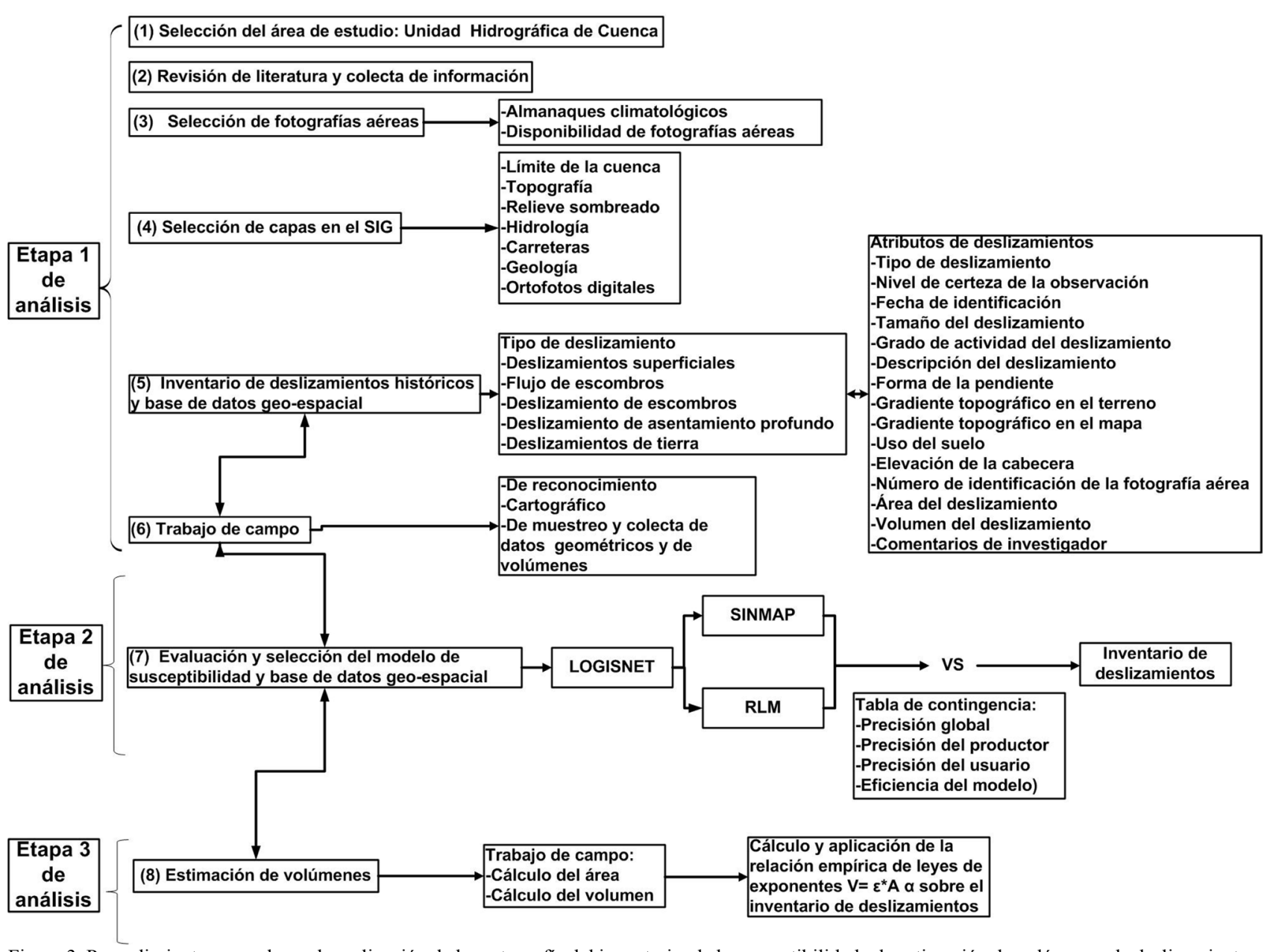

Figura 3. Procedimiento general para la realización de la cartografía del inventario, de la susceptibilidad y la estimación de volúmenes de deslizamientos.

1) para suelos volcánicos, con condiciones que asemejan a las del área de estudio (Selby, 1993; Legorreta-Paulín, 1999; Simon et al., 2000; Wawer y Nowocień, 2003; Simon y Collison, 2002).

Para el análisis de RLM, se utilizaron seis variables independientes en el análisis (altimetría, pendientes, curvatura del terreno, área de contribución de escurrimiento superficial, geología y uso del suelo). Estas variables fueron examinadas a través del Factor de Inflación de la Varianza (VIF) para diagnosticar su posible multicolinealidad (Pallant, 2005) y codificadas para ser usadas en el análisis (Tabla 2).

La Regresión Logística Múltiple fue calculada utilizando el método backward (retrospectivo), el cual se refiere a examinar las variables dentro del modelo para ver si alguna(s) de ellas debe(n) ser eliminada(s) del análisis. El modelo comienza con todas las variables independientes y las va probando una a una para ver cuál de éstas puede ser removida sin tener un efecto sustancial sobre el ajuste correcto que el modelo hace de los datos observados (Field,
2005). Una vez analizada la probabilidad de deslizamientos se asignó píxel por píxel al mapa de susceptibilidad con base en la función logística:

$1 /[1+\operatorname{Exp}-(-1.622+$ mapa altimétrico $(-3.5 \mathrm{E}-04)+$ mapa de pendientes $(0.120)+$ mapa de área de contribución de escurrimiento superficial (7.6E-07) + mapa de uso del suelo $(-0.151)+$ mapa de geología $(0.190)+$ mapa de curvatura (-0.433))], a través del uso del sistema LOGISNET (Legorreta-Paulín y Bursik, 2009) y del programa estadístico SPSS. Un esquema subjetivo de diez clases se utilizó para clasificar y mostrar la probabilidad de deslizamientos a intervalos de 0.1 (Figura 4c).

Para evaluar el desempeño de los modelos, se realizó un análisis cuantitativo por medio de una tabla de contingencia entre el mapa resultante de los modelos y el inventario de deslizamientos. La tabla de contingencia muestra la cantidad de superposición entre el inventario y los modelos, la cual se utilizó para calcular la precisión y la eficiencia de los modelos. Se usó: 1) la precisión del producto (Produce's accuracy) que representa la razón entre el número de 


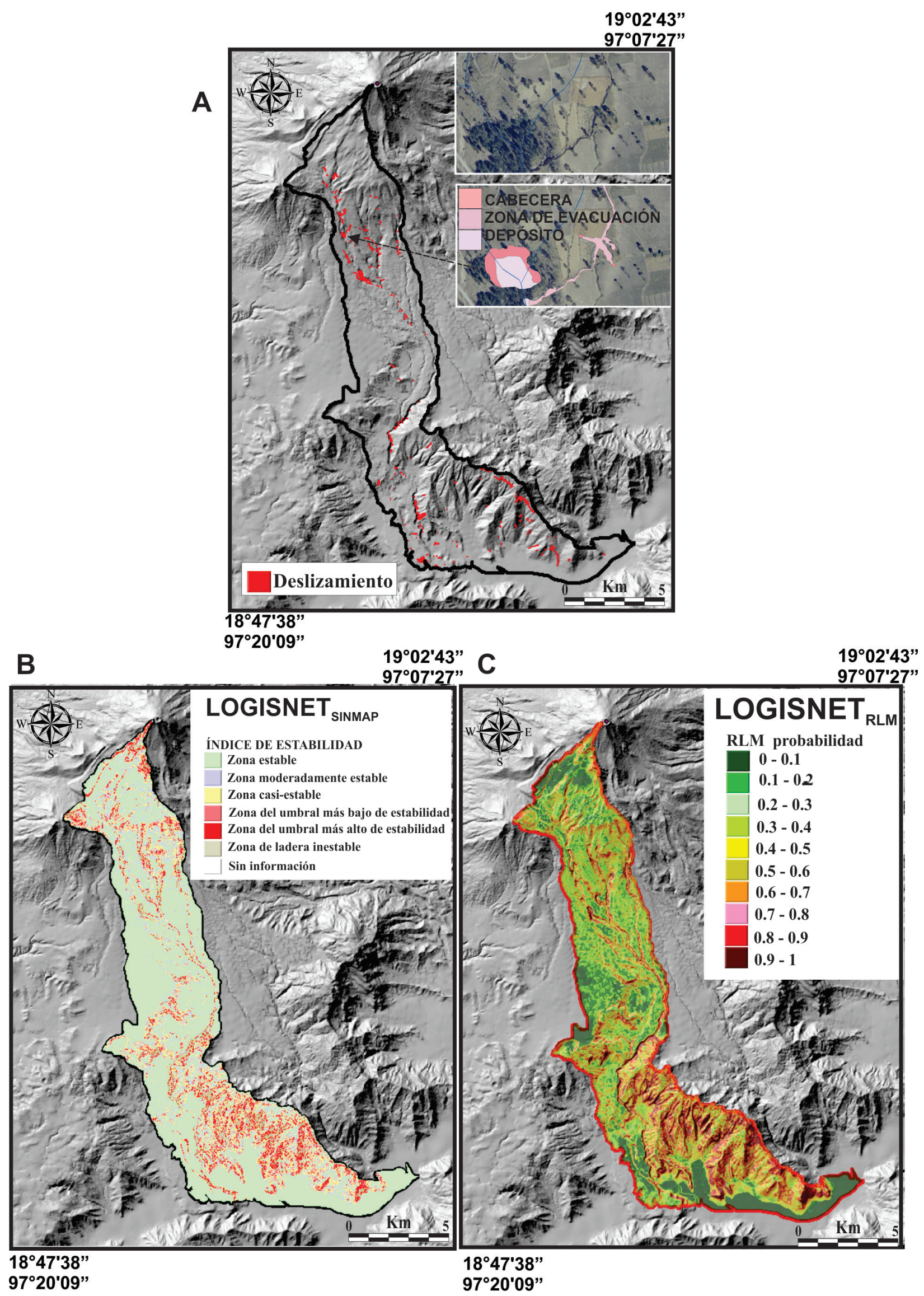

Figura 4. a) Inventario de deslizamientos; b) Modelo de susceptibilidad de SINMAP; c) Modelo de susceptibilidad de RLM. 
píxeles clasificados correctamente en cada categoría y el número total de píxeles verdaderos para esa categoría, 2) la precisión del usuario (User's accuracy), calculada como la razón entre el número de píxeles correctamente clasificados en cada categoría y el número total de píxeles que están clasificados por el modelo en esa categoría (Lilllesand y Kieffer, 2000), y 3) la eficiencia del modelo (Model efficiency), calculado como la razón del número de píxeles indicados correctamente como píxeles de deslizamiento, menos el número de píxeles indicados incorrectamente como píxeles de deslizamiento, entre el número total de los píxeles que representan verdaderos deslizamientos en

Tabla 1. Parámetros geotécnicos usados en SINMAP.

\begin{tabular}{lc}
\hline MDE & $10 \mathrm{~m}$ \\
Espesor del suelo $(\mathrm{m})$ & 1 \\
Gravedad $\left(\mathrm{m} / \mathrm{s}^{2}\right)$ & 9.81 \\
Densidad del agua $\left(\mathrm{kg} / \mathrm{m}^{3}\right)$ & 1000 \\
Densidad del suelo $\left(\mathrm{kg} / \mathrm{m}^{3}\right)$ & 1850 \\
T/R Min $(\mathrm{m})$ & 250 \\
T/R Max $(\mathrm{m})$ & 433.33 \\
Cohesión Min (sin unidades*) & 0.14 \\
Cohesión Max (sin unidades*) & 0.25 \\
Phi Min $\left(^{\circ}\right)$ & 30 \\
Phi Max $\left({ }^{\circ}\right)$ & 32 \\
Ponderación del usuario & 1 \\
\hline
\end{tabular}

* SINMAP requiere un factor de cohesión sin dimensión como entrada, que se obtiene dividiendo la cohesión $(\mathrm{Pa})$ por la gravedad $\left(\mathrm{m} / \mathrm{s}^{2}\right)$, la densidad del suelo $\left(\mathrm{kg} / \mathrm{m}^{3}\right)$ y espesor del suelo (m) (Pack et al., 1997).

Tabla 2. Variables y la codificación usada en la RLM.

\begin{tabular}{|c|c|}
\hline Índice & Variable \\
\hline & Elevación \\
\hline No codificada & Datos en crudo (sin clasificar) \\
\hline \multirow{3}{*}{ No codificada } & Ángulo de la pendiente \\
\hline & Datos en crudo (sin clasificar) \\
\hline & Curvatura de la pendiente \\
\hline-1 & Relieve cóncavo \\
\hline 0 & Relieve plano \\
\hline 1 & Relieve convexo \\
\hline \multirow{3}{*}{ No codificada } & Área de contribución de escurrimiento superficial \\
\hline & Datos en crudo (sin clasificar) \\
\hline & Geología \\
\hline 1 & Cuaternario (Aluvión) (Qal) \\
\hline 2 & Cuaternario (Brecha sedimentaria) (Qbs) \\
\hline 3 & Cuaternario (Toba volcánica félsica) (QTa) \\
\hline 4 & Cenozoico Superior (Andesita) (TsA) \\
\hline 5 & Cenozoico Superior (Toba volcánica intermedia) (TsTi) \\
\hline 6 & Cenozoico Superior (Brecha volcánica intermedia) (TsBvi) \\
\hline \multirow[t]{2}{*}{7} & Cretácico Superior (Caliza) (Kscz) \\
\hline & Uso del suelo \\
\hline 1 & Agricultura de subsistencia \\
\hline 2 & Pastoreo \\
\hline 3 & Pastizal alpino \\
\hline 4 & Bosque de oyamel (Abies religiosa) \\
\hline 5 & Bosque de pino \\
\hline 6 & Bosque de pino-encino \\
\hline 7 & Bosque de encino \\
\hline
\end{tabular}

el inventario correspondiente. La eficiencia del modelo tiene un valor máximo de 1 cuando el modelo ha indicado todos los deslizamientos de tierra correctamente, y tiene un valor negativo cuando el número de deslizamientos incorrectamente indicados por el modelo, es mayor que el número de deslizamientos correctamente predicho (Van Den Eeckhaunt et al., 2005).

Para la tercera etapa del trabajo de campo, se midió el área y volumen de 102 cicatrices de deslizamientos, a fin desarrollar una relación teórica volumen-área de deslizamientos superficiales en la cuenca; aquí se excluyó el volumen de depósitos o el área de evacuación del deslizamiento. La geometría de los deslizamientos se midió con cinta, estadal y distanciómetro láser. La profundidad del deslizamiento en la cabecera y en las paredes laterales a lo largo de transectos, así como la distancia horizontal a lo largo de los transectos, fueron utilizados para estimar el volumen del deslizamiento. Las cicatrices que fueron sustancialmente reforestadas o que se estimó que fueron cubiertas por vegetación por más de 17 años, no fueron incluidas en la cartografía del inventario. Las medidas del área de deslizamientos $(A)$ y el volumen $(V)$ se utilizó para establecer y alimentar una relación empírica que tomó la forma de una ley de potencia:

$V=\varepsilon^{*} A^{\alpha}$,

(Korup et al., 2004; Kalderon-Asael et al., 2008; Guzzetti et al., 2009), con un exponente de escala de $\alpha=$ 1.004. Esta relación aplicada al área de los deslizamientos cartografiados en el inventario se utilizó para estimar el volumen total de material potencial que los deslizamientos pueden aportar al cauce.

\section{Resultados}

El trabajo de campo y la fotointerpretación permitieron cartografiar 442 deslizamientos. Con este inventario multitemporal expresado como mapa, se pudo observar que los deslizamientos superficiales que incluyen flujos de escombros y deslizamientos de escombros (de menos de 2 $\mathrm{m}$ de profundidad), es el tipo de proceso predominante por número $(91.4 \%)$, sobre los deslizamientos de caída de rocas (7.5\%) y los deslizamientos rotacionales de asentamiento profundo y flujos de tierra (1.1\%). Sin embargo, no es así por área de afectación. Con la sobreposición entre el inventario y el mapa de geología, se pudo establecer que del total de deslizamientos, el $66.3 \%$ se encuentra en rocas o depósitos volcánicos, el 33\% en caliza y lutita y el $0.7 \%$ en depósitos sedimentarios. En la parte alta de la cuenca, los flujos de lava andesíticos facilitan el desarrollo de caída de rocas más que deslizamientos superficiales (Figura 2b). En la parte media de la cuenca, donde existen depósitos de caída de ceniza volcánica y de flujos piroclásticos, son los deslizamientos superficiales y de asentamiento profundo los procesos predominantes (Figura 2c). Además, se presentan procesos gravitacionales de caída de roca a lo largo del 
cauce principal, donde flujos de lava forman escarpes. En la parte baja de la cuenca tienden a ocurrir flujos de tierra y deslizamientos de escombros en caliza y lutita intemperizada (Figura 2d).

Los resultados obtenidos por ambos modelos de análisis de la susceptibilidad, muestran, desde un punto de vista cualitativo, una alta inestabilidad a lo largo de las zonas montañosas volcánicas y sedimentarias y a lo largo de las paredes del valle de pendientes fuertes. La sobreposición y grado de similitud entre el inventario de deslizamientos y la predicción de los modelos no es perfecta, y existe claramente una sobreestimación de las áreas afectadas por deslizamientos en ambos modelos (Figura 4). Sin embargo, la sobrepredicción se da en áreas que tienen las condiciones necesarias para deslizarse en el futuro, como se pudo observar durante el trabajo de campo. Cuantitativamente, la eficiencia de SINMAP y RLM, se evaluó en términos de precisión del productor, la precisión del usuario y la eficiencia del modelo (Tabla 3). La precisión del productor muestra que la predicción de la RLM tiene mejor coincidencia $(72.33 \%$ ) con el mapa del inventario de deslizamientos en comparación con SINMAP (45.39\%). Ambos modelos tienen un bajo porcentaje de precisión de usuario y valores negativos en la prueba de eficiencia del modelo debido a su sobrepredicción. Ésta conduce a que más zonas sin deslizamientos sean clasificadas incorrectamente como zonas de deslizamiento en el modelo.

Con base en el análisis detallado de la relación áreavolumen de 102 deslizamientos medidos en el campo, se calculó la ley de potencias para los deslizamientos igual a:$$
V=0.7908 * A^{1.004}
$$

Ésta se aplicó dentro del SIG para el área remanente de la población de deslizamientos que no participaron en la formulación de dicha ley. El resultado muestra que los deslizamientos superficiales ubicados en el río principal tienen el potencial de aporte de $226584.08 \mathrm{~m}^{3}$ de material al cauce.

\section{Discusión y conclusiones}

Este trabajo presenta y examina la implementación de un método para la creación de inventarios multitemporales y la evaluación de susceptibilidad a deslizamientos en terrenos volcánicos inestables. Este método se basa en la estandarización y la integración de capas temáticas y sus bases de datos geoespaciales correspondientes dentro de un SIG.

Al abordar directamente la metodología para la cartografía de deslizamientos, las autoridades locales, como las Direcciones Estatales de Protección Civil de los estados de Puebla y Veracruz y otras organizaciones gubernamentales se beneficiarán con respecto a la mitigación y a la prevención del riesgo de deslizamientos. Se hace énfasis en el hecho de que esta investigación es la primera que se hace en el área de estudio para desarrollar una
Tabla 3. Precisión del productor, precisión del usuario y eficiencia del modelo.

\begin{tabular}{lcc}
\hline & LOGISNET $_{\text {SINMAP }}$ & $\begin{array}{c}\text { LOGISNET }_{\text {RLM }} \\
\text { (Método backward })\end{array}$ \\
\hline Precisión del productor & Área de deslizamiento & Área de deslizamiento \\
Precisión del usuario & $45.39 \%$ & $72.33 \%$ \\
Eficiencia del modelo & $0.56 \%$ & $0.42 \%$ \\
\hline
\end{tabular}

metodología SIG más robusta y sistemática en la creación de inventarios, análisis de la susceptibilidad y de estimación de volúmenes de deslizamientos. Por lo tanto, está sujeta a adaptación, modificación y mejoramiento, conforme se validen los mapas y bases de datos geoespaciales en la cuenca, y se realicen nuevas evaluaciones y comparación con otras cuencas en México. Conocemos la limitación técnica del inventario de deslizamientos, cuya calidad depende de las habilidades y experiencia de los investigadores, la complejidad del área de estudio, así como la confiabilidad de la información disponible, incluyendo las fotografías aéreas para identificar los deslizamientos. Así por ejemplo, no se utilizaron almanaques climáticos ni registros pluviales para seleccionar las fotografías aéreas de épocas de lluvias o de sequía más adecuadas a utilizarse en el análisis. Esto obedeció a la escasa disponibilidad de fotografías aéreas en el área de estudio.

En este trabajo se introdujo la aplicación de SINMAP y RLM al analizarlos deslizamientos bajo condiciones naturales a fin de esbozar las ventajas y limitaciones de dichos modelos. Ambos poseen un moderado grado de concordancia con el inventario de deslizamientos. Los resultados de la validación del modelo ilustran que SINMAP y RLM tiende a la sobrepredicción, misma que se puede justificar en una evaluación de deslizamientos a escala regional debido a cuatro razones principales: en primer lugar, las zonas de deslizamientos sobrepredichas se encuentran por encima o cerca de los deslizamientos existentes, por lo tanto, estas zonas pueden tener el potencial para futuros deslizamientos; en segundo lugar, los deslizamientos predichos por los modelos y los existentes en el inventario de deslizamientos no tienen por qué coincidir, ya que los modelos son sólo una guía para evaluar las áreas de alta susceptibilidad a deslizarse; tercero, el inventario de deslizamientos puede no mostrar alguno, debido a que el tiempo en que fue construido no refleja futuras zonas inestables, por lo que hay una disimilitud entre el resultado del modelo y el inventario de deslizamientos, y cuarto, en el análisis de los modelos, el uso del modelo digital de elevación a $10 \mathrm{~m}$ de resolución y la generalización cartográfica de los mapas $1: 250000$ y 1:50000 para el análisis de SINMAP y RLM también es una limitante, ya que en esta resolución y generalización todos los tipos de deslizamiento tienden a perder la representación cartográfica y es difícil entrenar al modelo para separar áreas de deslizamiento de otras sin deslizamientos. Un 
análisis de susceptibilidad con más precisión y detalle quizás se obtendrá en situaciones donde la resolución de datos topográficos sea mejor, como la obtenida a través de la tecnología LIDAR. Sin embargo, aún no está disponible en muchas zonas remotas de México. Se considera más apropiado el uso de la RLM para futuros análisis, ya que tiene la posibilidad de incluir otras variables temáticas con las que puede mejorar su rendimiento en comparación con SINMAP. También la disponibilidad y empleo de un modelo digital de elevación de mayor resolución podría, a futuro, mejorar la predicción del modelo. No es posible comparar los resultados obtenidos en esta investigación con otros trabajos en los que se modela y compara SINMAP y RLM (Legorreta-Paulín y Bursik, 2008) por tratarse de condiciones locales distintas y en los que se usaron otras variables para analizar la susceptibilidad. A fin de corroborar los resultados de este trabajo es necesario, a futuro, utilizar el mismo análisis en ambos modelos, con las mismas variables y en cuencas con condiciones geológicas y ambientales similares.

La estimación de volúmenes aportados al cauce principal es un paso importante en la prevención de desastres en el área de estudio. Sin embargo, se hace hincapié en el hecho que la ley de potencias usada y el cálculo de volumen se realizó únicamente para el río principal y no incluye a los tributarios de toda la cuenca, ni todos los tipos de deslizamientos, ni al área de evacuación y depósitos, por lo que el volumen calculado está muy por debajo del volumen potencial total que los deslizamientos pueden aportar en eventos extraordinarios. Para realizar el cálculo del volumen aportado por los deslizamientos se consideró solamente la cabecera, pues es la fuente principal de aporte de material. Las zonas de evacuación y de depósito no se consideraron por ser afectadas por otros procesos distintos a los que controlan los deslizamientos. Así por ejemplo, la zona de evacuación, no sólo la gravedad -que es el factor que controla los deslizamientos-, sino también procesos fluviales actúan en el área, lo que hace imposible la separación y cuantificación de material aportado por deslizamientos laterales en el cauce.

Es también necesario considerar que el volumen calculado es una estimación del material que potencialmente podría ser desplazado hacia el cauce en un evento extraordinario, en el que todos los deslizamientos estén activos y desplazando la totalidad de su escarpe. Esta situación no fue observada en su totalidad en campo; en cambio, en algunos deslizamientos se observó que el material de la cabecera se desplazó totalmente y en otros parcialmente durante la época de lluvias.

Pese a las limitaciones mencionadas, el inventario elaborado, el análisis de la susceptibilidad y la estimación de volúmenes es la base de una metodología integral para gestionar y apoyar los estudios de pronóstico de inestabilidad de laderas.

A futuro, la investigación considerará el modelizado de volúmenes aportados por tipo de deslizamiento, la caracterización de los procesos gravitacionales por formas del terreno, la inclusión de otras variables temáticas o la actualización de las existentes, así como la obtención del mapa de riesgo.

\section{Agradecimientos}

Los autores agradecen a las autoridades del Departamento de Geología de la Universidad de Buffalo, Nueva York, al Consorcio Internacional sobre Deslizamientos (ICL), a las Divisiones de Prácticas Forestales y de Geología y Recursos Naturales del Departamento de Recursos Naturales (DNR) del Estado de Washington, así como al laboratorio de Análisis Geoespacial del Instituto de Geografía de la UNAM por su aprobación y ayuda. Esta investigación fue financiada por el Programa de Apoyo a Proyectos de Investigación e Innovación Tecnológica (PAPIIT), UNAM No. IB100412RR180412).

\section{Referencias}

Ayalew, L., Yamagishi, H., 2005, The application of GIS-based logistic regression for landslide susceptibility mapping in the Kakuda-Yahiko Mountains, Central Japan: Geomorphology, 65, 15-31.

Bai, S.B., Wang,J., Lü, G.N., Zhou, P.G., Hou, S.S., Xu, S.N., 2010, GIS-based logistic regression for landslide susceptibility mapping of the Zhongxian segment in the Three Gorges area, China: Geomorphology, 115, 23-31.

Beven, K.J., Kirkby, M.J., 1979, A physically based variable contributing area model of basin hydrology: Hydrological Sciences Bulletin, 24, 43-69.

Blahut, J., Van Westen, C.J., Sterlacchini, S., 2010, Analysis of landslide inventories for accurate prediction of debris-flow source areas: Geomorphology, 119(1-2), 36-51.

Borga, M., Fontana, G.D., Cazorzi, F., 2002a, Analysis of topographic and climatic control on rainfall-triggered shallow landsliding using a quasi-dynamic wetness index: Journal of Hydrology, 268, 56-71.

Borga, M., Fontana, G.D., Gregoretti, C., Marchi, L., 2002b, Assessment of shallow landsliding by using a physically based model of hillslope stability: Hydrological Processes, 16, 2833-2851.

Can, T., Nefeslioglu, H.A., Gokceoglu, C., Sonmez, H., Duman, T.Y., 2005, Susceptibility assessments of shallow earth flows triggered by heavy rainfall at three catchments by logistic regression analyses: Geomorphology, 72, 250-271.

Capra, L., Lugo-Hubp, J., 2006, Fenómenos de remoción en masa en el poblado de Zapotitlán de Méndez, Puebla: Relación entre litología y tipo de movimiento: Revista Mexicana de Ciencias Geológicas, 20(2), 95-106.

Capra, L., Macías, J.L., Scott, K.M., Abrams, M., Garduño-Monroy, V.H., 2002, Debris avalanches and debris flows transformed from collapses in the Trans-Mexican Volcanic Belt, México. Behavior, and implication for hazard assessment: Journal of Volcanology and Geothermal Research, 113(1-2), 81-110.

Capra, L., Lugo-Hubp, J., Zamorano, J.J., 2006, La importancia de la Geología en el estudio de los procesos de remoción en masa: el caso de Totomoxtla, Sierra Norte de Puebla, México: Boletín de la Sociedad Geológica Mexicana, 58(2), 205-214.

Carrasco-Núñez, G., Díaz-Castellón, R., Siebert, L., Hubbard, B., Sheridan, M.F., Rodríguez, S.R., 2006, Multiple edifice-collapse events in the Eastern Mexican Volcanic Belt: the role of sloping substrate and implications for hazard assessment:Journal of Volcanology and Geothermal Research, 158, 151-176. 
Castellanos-Abella, E.A., Van Westen, C.J., 2008, Qualitative landslide susceptibility assessment by multicriteria analysis: a case study from San Antonio del Sur, Guantánamo, Cuba: Geomorphology 94, 453-466.

Centro Nacional de Prevención de Desastres (CENAPRED), 2004, Guía básica para la elaboración de mapas estatales y municipales de peligros de riesgos: Centro Nacional de Prevención de Desastres, Secretaría de Gobernación, México, 389 p.

Chinnayakanahalli, K., Tarboton, D.G., Pack, R.T., 2003, An objective method for the intercomparison of terrain stability models: Eos Transaction, American Geophysical Union, Fall Meeting Supplement, 84(46), Abstract H31C-0480.

Clerici, A., Perego, S., Tellini, C., Vescovi, P., 2002, A procedure for landslide susceptibility zonation by the conditional analysis method: Geomorphology, 48, 349-364.

Concha-Dimas, A., Cerca, M., Rodríguez-Elizarrarás, S., Watters, R.J., 2005, Geomorphological evidence of the influence of preexisting basement structure on emplacement and deformation of volcanic edifices at the Cofre de Perote-Pico de Orizaba chain and implications for avalanche generation: Geomorphology, 72, 19-39.

Cruden, D.M., Varnes, D., 1996, Landslide types and processes, en Turner, A.K., Shuster, R.L. (eds.), Landslides: Investigation and Mitigation: Transportation Research Board, Special Report, 247, 36-75.

Dai, F.C., Lee, C.F., Ngai, Y.Y., 2002, Landslide risk assessment and management: an overview: Engineering Geology, 64, 65-87.

De la Cruz-Reyna, S., Carrasco-Núñez, G., 2002, Probabilistic hazard analysis of Citlaltépetl (Pico de Orizaba) Volcano, eastern Mexican Volcanic Belt:Journal of Volcanology and Geothermal Research, 113, 307-318.

Deb, S.K., El-Kadi, A.I., 2009, Susceptibility assessment of shallow landslides on Oahu, Hawaii, under extreme-rainfall events: Journal of Geomorphology, 108, 219-233, doi:10.1016/j. geomorph.2009.01.009

Demoulin, A., Chung, C.F., 2007, Mapping landslide susceptibility from small datasets: a case study in the Pays de Herve (E Belgium): Geomorphology, 89, 391-404.

Dietrich, W.E., Bellugi, D., Real de Asua, R., 2001,Validation of the shallow landslide model, SHALSTAB, for forest management, en Wigmosta, M.S, Burges, S.J. (eds.), Land Use and Watersheds: Human influence on hydrology and geomorphology in urban and forest areas: American Geophysical Union, Water Science and Application, 2, 195-227.

Evans, S.G.,Scarascia-Mugnozza, G., Strom, A.L., Hermanns, R.L., Ischuk, A., Vinnichenko, S., 2006, Landslides from massive rock slope failure and associated phenomena, en Evans, S.G., ScarasciaMugnozza, G., Strom, A.L., Hermanns, R.L. (eds.), Landslides from massive rock slope failure: Dordrecht, Springer, NATO Science Series IV,49, 3-52.

Field, A., 2005, Discovering Statistics Using SPSS for Windows: Advanced Techniques for Beginners (Introducing Statistical Methods): Thousand Oaks, California, Second Ed., Sage Publications, 816 p.

García-Palomo, A., Carlos-Valerio, V., López-Miguel, C., GalvánGarcía,A., Concha-Dimas, A., 2006, Landslide inventory map of Guadalupe Range, north of the Mexico Basin: Boletín de la Sociedad Geológica Mexicana, 58(2), 195-204.

Guzzetti, F., Ardizzone, F., Cardinali, M., Rossi, M., 2009, Landslide volumes and landslide mobilization rates in Umbria, central Italy: Earth and Planetary Science Letters, 279, 22-229.

Hammond, C., Hall, D., Miller, S., Swetik, P., 1992, Level I Stability Analysis (LISA) documentation for version 2.0: General Technical Report INT-285, United States Department of Agriculture, Forest Service, Intermountain Research Station, Ogden, Utah, 190 p.

Haneberg, W.C., 2005, New quantitative landslide hazard assessment tools for planners, en Schwab, J.C., Gori, P.L., Jeer, S. (eds.), Landslide Hazards and Planning: Planning Advisory Service Report Number 533/534, American Planning Association, 76-84.

Hervás, J., Bobrowsky, P., 2009, Mapping: Inventories, Susceptibility,
Hazard and Risk, en Sassa, K.,Canuti, P. (eds.), Landslides - Disaster Risk Reduction: Springer, Berlin, 321-349.

Hubbard, B.E., 2001, Volcanic Hazard Mapping Using Aircraft, Satellite and Digital Topographic Data: Pico de Orizaba (Citlaltépetl), México: Buffalo, New York, State University of New York. Teisis de Doctorado, $708 \mathrm{p}$.

Hubbard, B.E, Sheridan, M.F., Carrasco-Nunez, G., Díaz-Castellón, R., Rodríguez, S., 2007, Comparative lahar hazard mapping at Volcán Citlaltépetl, Mexico, using SRTM, ASTER and DTED-1 Digital Topography: Journal of Volcanology and Geothermal Research, 160(1), 99-124.

Kalderon-Asael, B., Katz, O., Aharonov, E., Marco, S., 2008, Modeling the relationship between areaand volume of landslides: Geological Survey of Israel, Report GSI/06/2008, 1-16.

Kleinbaum, D.G., Klein, M., 2002,Logistic Regression: A Self-learning Text: New York, Springer, Second edition, $513 \mathrm{p}$.

Korup, O., McSaveney, M.J., Davies, T.R., 2004, Sediment generation and delivery from large historic landslides in the Southern Alps, New Zealand: Geomorphology, 61(1/2), 189-207.

Lan, H.X., Zhou, C.H., Wang, L.J., Zhang, H.Y., Li, R.H., 2004, Landslide hazard spatial analysis and prediction using GIS in the Xiaojiang watershed, Yunnan, China: Engineering Geology, 76, 109-128.

Legorreta-Paulín, G., 1999, Procesos geomorfológicos en el volcán Paricutín: México, D.F., Universidad Nacional Autónoma de México, tesis de maestría, $195 \mathrm{p}$.

Legorreta-Paulín, G., Bursik, M., 2008, Logisnet: A tool for multimethod, multiple soil layers slope stability analysis: Computers and Geosciences, computers \& geosciences. 35(5), 1007-1016, doi:10.1016/j.cageo.2008.04.003.

Legorreta-Paulín, G., Bursik, M., 2009, Assessment of landslides susceptibility: LOGISNET: a tool for multimethod, multilayer slope stability analysis, Ed. Verlag Dr. Müller, USA, 360 p.

Legorreta-Paulín, G., Bursik, M., Lugo-Hubp, J., Zamorano-Orozco, J.J., 2010, Effect of pixel size on cartographic representation of shallow and deep-seated landslide, and its collateral effects on the forecasting of landslides by SINMAP and Multiple Logistic Regression landslide models: Physics and Chemistry of the Earth, 35, 137-148.

Lilllesand, T.M., Kieffer, R.W., 2000, Remote Sensing and image interpretation: New York, John Wiley \& Sons, 4th ed.,724 p.

Macías, J.L., 2005, Geología e historia eruptiva de algunos de los grandes volcanes activos de México: Boletín de la Sociedad Geológica Mexicana, Volumen Conmemorativo del Centenario, LVII(3), 379-424.

Meisina, C., Scarabelli, S., 2007, A comparative analysis of terrain stability models for predicting shallow landslides in colluvial soils: Geomorphology, 87, 207-223.

Metternicht, G., Hurni, L., Gogu, R., 2005, Remote sensing of landslides: an analysis of the potential contribution to geo-spatial systems for hazard assessment in mountainous environments: Remote Sensing of Environment, 98, 284-303.

Montgomery, D.R., Dietrich, W.E., 1994, A physical based model for the topographic control on shallow landslides: Water Resources Research, 30, 1153-1171.

Morrissey, M.M., Wieczorek, G.F., Morgan, B.A., 2001, A comparative analysis of hazard models for predicting debris flows in Madison County, Virginia: United States Geological Survey Open-File Report 01-0067, 17p., disponible en $<$ http://pubs.usgs.gov/of/2001/ofr-010067/ofr-01-0067.html>.

Ohlmacher, G.C., Davis, J.C., 2003, Using multiple logistic regression and GIS technology to predict landslide hazard in northeast Kansas, USA: Engineering Geology, 69, 331-343.

O'Loughlin, E.M., 1986, Prediction of surface saturation zones in natural catchments by topographic analysis: Water Resources Research, $22,794-804$

Pack, R.T.,Tarboton, D.G., Goodwin, C.N., 1997, SINMAP- A Stability Index Approach to Terrain Stability Hazard Mapping, Users Manual, 75p., disponible en $<\mathrm{http}: / /$ hydrology.neng.usu.edu/sinmap/> 
Pack, R.T., Tarboton, D.G., Goodwin, C.N., 1998, The SINMAP approach to terrain stability mapping: Proceedings of the Eighth Congress of the International Association of Engineering Geology, Vancouver, British Columbia, Canada, disponible en <http://hydrology.neng. usu.edu/sinmap/>.

Pack, R.T., Tarboton, D.G., Goodwin, C.N., 2001, Assessing terrain stability in a GIS using SINMAP: Proceedings of the 15th Annual GIS Conference, Vancouver, British Columbia, disponible en $<\mathrm{http}: / /$ hydrology.neng.usu.edu/sinmap/>.

Palacios, D., Parrilla, G., Zamorano, J.J., 1999, Paraglacial and postglacial debris flows on Little Ice Age terminal moraine: Jamapa Glacier, Pico de Orizaba (Mexico): Geomorphology, 28, 95-118.

Pallant, J., 2005, SPSS survival manual: a step by step guide to data analysis using SPSS for Windows (Version 12): Buckingham, Open University Press, $319 \mathrm{p}$.

Peark, M.R., Ng, K.Y., Zhang, D.D., 2005, Landslide and sediment delivery to a drainage system: some observations from Hong Kong: Journal of Asian Earth Science, 25, 821-836.

Pérez-Gutiérrez, R., 2007, Análisis de la vulnerabilidad por los deslizamientos en masa: caso Tlacuitlapa, Guerrero: Boletín de la Sociedad Geológica Mexicana, 59(2), 171-181.

Pringle, P.T., Brunego, M.J., 2004, Landslide zonation project, Bacon Creek watershed, Skagit County, Washington: Washington Department of Natural Resources, 11p., disponible en <http://www.dnr.wa.gov/ BusinessPermits/Topics/LandslideHazardZonation/Pages/fp_lhz completed.aspx>, consultado 1 de febrero de 2009.

Rodríguez, S., Mora, I., Murrieta, J., 2006, Flujos de baja concentración asociados con lluvias de intensidad extraordinaria en el flanco sur del volcán Pico de Orizaba (Citlaltépetl), México: Boletín de la Sociedad Geológica Mexicana, Número Especial de Geología Urbana 2, 223-236.

Rodríguez, S., Mora, I., Murrieta, J., Morales, B.W.V., 2011, Peligros geológicos más frecuentes en el estado de Veracruz: Xalapa, Veracruz, Universidad Veracruzana, $85 \mathrm{p}$.

Secretaría de Protección Civil, 2010, Atlas de peligros geológicos e hidrometeorológicos del estado de Veracruz, en Mora González, I., Morales Barrera, W., Rodríguez Elizarrarás, S., (comp.), Secretaría de Protección Civil del estado de Veracruz: Universidad Veracruzana, UNAM. IV. 39 mapas, escalas varias, Atlas en proceso de publicación.

Selby, M.J., 1993, Hillslope materials and process: Oxford, Oxford University Press, $451 \mathrm{p}$.

Sharikahn, I.Y., Walsh ,T., 2007, Mason Watershed, Landslide hazard zonation project, Mason County, Washington: Washington Department of Natural Resources, 138 p., disponible en $<\mathrm{http}$ ://www. dnr.wa.gov/BusinessPermits/Topics/LandslideHazardZonation/ Pages/fp_lhz_completed.aspx>, consultado 1 de febrero de 2009.

Sheridan, M., Carrasco-Núñez, G., Hubbard, B., Siebe, C., Rodríguez, S., 2002, Mapa de peligros volcánicos del Volcán Citlaltépetl (Pico de Orizaba), Escala 1:250000: UNAM, Gobiernos de los Eestados de Puebla y Veracruz.

Siebe, C., Abrams, M., Macías, J.L., 1996, Derrumbes gigantes, depósitos de avalanchas de escombros y edad del actual cono del volcán Popocatépetl, en Volcán Popocatépetl, Capítulo V Aspectos Geológicos, Estudios Realizados Durante la Crisis de 19941995: Sistema Nacional de Protección Civil, Centro Nacional de Prevención de Desastres, Universidad Nacional Autónoma de México, 195-220.
Simon, A., Collison, A.J.C., 2002,Quantifying the mechanical and hydrologic effects of riparian vegetation on streambank stability: Earth Surface Processes and Landforms, 27, 527-546.

Simon, A., Curini, A., Darby, S.E., Langendoen, E.J., 2000, Bank and nearbank processes in an incised channel: Geomorphology, 35, 193-217.

Van Den Eeckhaunt, M., Poesen, J., Verstraeten, G., Vanacker, V., Moeyersons, J., Nyssen, J., Van Beek, L.P.H., 2005, The effectiveness of hillshade maps and expert knowledge in mapping old deep-seated landslides: Geomorphology, 67, 351-363.

Washington State Department of Natural Resources (DNR), Forest Practices Division, 2006, Landslide Hazard Zonation (LHZ) Mapping Protocol, version 2.0, diponible en $<\mathrm{http}$ ://www.dnr. wa.gov/BusinessPermits/Topics/LandslideHazardZonation/Pages/ fp $l$ lhz review.aspx $>$.

Wawer, R., Nowocień, E., 2003, Application of SINMAP terrain stability model in Grodarz stream watershed: Electronic Journal of Polish Agricultural Universities, 6(1), disponible en <http://www.ejpau. media.pl/volume6/issue1/environment/abs-03.html>.

Wenkey, D., Jen C.H.,Böse, M., Lin, J.C., 2011, Assessment of sediment delivery from successive erosion on stream-coupled hillslopes via a time series of topographic surveys in the central high mountain range of Taiwan: Quaternary International, 263, 14-25, doi:10.1016/j. quaint.2011.02.018.

Wieczorek, G.F., 1984, Preparing a detailed landslide inventory map for hazard evaluation and reduction: Bulletin of the Association of Engineering Geology, 21, 337-342.

Winsemius, H.C., Savenije, H.H.G., Gerrits, A.M.J., Zapreeva, E.A., Klees, R., 2005, Comparison of two model approaches in the Zambezi river basin with regard to model reliability and identifiability: Hydrology and Earth System Sciences Discussions, 2, 2625-2661.

Yesilnacar, E., Topal, T., 2005, Landslide susceptibility mapping: A comparison of logistic regression and neural networks methods in a medium scale study, Hendek region (Turkey): Engineering Geology, 79, 251-266.

Zaitchik, B.F., Van Es, H.M., Sullivan, P.J., 2003, Modeling Slope Stability in Honduras: Parameter Sensitivity and Scale of Aggregation:Soil Science Society of America Journal, 67, 268-278.

Zimbelman, D.R., Watters, R.J., Firth, I.R., Breit, G.N., Carrasco-Núñez, G., 2004, Stratovolcano stability assessment methods and results from Citlaltépetl, Mexico: Bulletin of Volcanology, 66, 66-79

Zhou, G., Esaki, T., Mitani, Y., Xie, M., Mori, J., 2003, Spatial probabilistic modeling of slope failure using an integrated GIS Monte Carlo simulation approach: Engineering Geology, 68, 373-386.

Manuscrito recibido: Junio 20, 2013.

Manuscrito corregido recibido: Noviembre 22, 2013.

Manuscrito aceptado: Noviembre 20, 2013. 